\title{
Starspot activity on the RS CVn-type binary II Pegasi during 1999-2001
}

\author{
S.-H. Gu, H.-S. Tan, X.-B. Wang, and H.-G. Shan
}

\author{
National Astronomical Observatories/Yunnan Observatory, Joint Laboratory for Optical Astronomy, Chinese Academy \\ of Sciences, Kunming, PR China
}

Received 19 September 2002 / Accepted 6 May 2003

\begin{abstract}
By means of Doppler imaging, new high-resolution spectroscopic observations of II Peg, carried out in three observing seasons from 1999 to 2001, were analyzed. The lines FeI6430.844 $\AA$ and CaI6439.075 $\AA$ were chosen as map lines, and three surface maps of II Peg were obtained. The results demonstrate that the main features of these surface maps are highlatitude active regions around $60^{\circ}$ and no polar cap is seen in the maps. The surface patterns of II Peg show large changes, the largest scale active regions appear in July-Aug. 1999 and then they become small and separated in Feb. 2000. The active longitude switches to another hemisphere in Nov.-Dec. 2001 from the one in Feb. 2000. Such spot evolution is not consistent with the activity cycle derived previously.
\end{abstract}

Key words. stars: late-type - stars: activity - stars: imaging - stars: starspots - stars: individual: II Peg

\section{Introduction}

II Peg is one of the most active RS CVn-type binaries. The main features of such a star are significant photometric variability (Rodonò et al. 2000), long-term starspot activity (Berdyugina et al. 1998a, 1999a; Rodonò et al. 2000), very strong $\mathrm{H}_{\alpha}$ emission above continuum spectrum, CaII H\&K, IRT core emission and optical flares (Rucinski 1977; Montes et al. 1997; Berdyugina et al. 1999b; Gu \& Tan 2001).

II Peg is a single-lined spectroscopic binary, the active primary is a K2IV type subgiant, and the unseen secondary is a low-mass M-dwarf (Berdyugina et al. 1998b). Because it is a very active system, and there are many activity phenomena visible, II Peg has attracted much interest. In order to study the evolution of solar-like activity on II Peg, we set up a longterm monitoring project in 1999 . The preliminary results in 2000-2001 were reported by us (Gu et al. 2002).

Berdyugina et al. (1998a, 1999a) derived a spot activity cycle of 9.3 years from their 7 year surface imaging results, and proposed that the "flip-flop" phenomenon can be a tracer of spot activity. But, based on our new results in this paper, it seems that the spot activity is more complex than previous thought, and we have found that active region evolution does not obey the activity cycle obtained by Berdyugina et al. (1998a, 1999a).

Here we present new results of Doppler imaging of II Peg derived using the data from our 1999-2001 observing seasons. In Sect. 2, we describe the spectroscopic observations and data

Send offprint requests to: $\mathrm{S} . \mathrm{-H}$. Gu, e-mail: shenggu@public.km.yn.cn reduction. The Doppler imaging is given in Sect. 3. In Sect. 4, the results are discussed and compared with previous surface imaging results. The summary is given in Sect. 5 .

\section{Observation and data reduction}

The new spectroscopic observations of II Peg were made using the Coude echelle spectrograph (Jiang 1996; Zhao \& Li 2001) on the $2.16 \mathrm{~m}$ telescope at the Xinglong station of the National Astronomical Observatories from July 26 to August 1, 1999, February 19 to 24, 2000, and November 23 to December 1, 2001. The spectrograph is equipped with a $1024 \times 1024$ pixel Tektronix CCD detector, and its resolution power is about 34000 . In the spectral region including

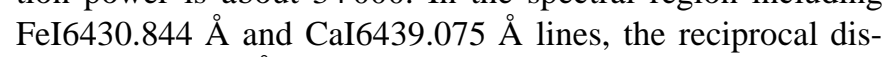
persion is $0.089 \AA$ per pixel. The $F W H M$ of the arc comparison lines is $0.18 \AA$ which is very close to the resolution power of the spectrograph. The stability of the flat field over time is about $0.8 \%$ during our observations. In the selected spectral region, the $S / N$ ratio per pixel for a typical exposure is about 70 . The observing log is listed in Table 1, where the Heliocentric Julian dates, orbital phases, exposure times, and $S / N$ ratios of the spectra are given. The orbital phases of the observations are calculated according to the orbital ephemeris of Berdyugina et al. (1998b).

All observational data had been reduced using IRAF package in the standard fashion, including image trimming, bias subtraction, flat-field division, spectrum extraction, and cosmic ray removal. The wavelength calibration was obtained by taking spectra of a Th-Ar lamp. Finally, all spectra had been normalized using a cubic spline fit to the observed continuum. 
Table 1. The spectroscopic observing log of II Peg.

\begin{tabular}{|c|c|c|c|}
\hline JD(Hel.) & Phase & Exp. time (s) & $S / N$ \\
\hline \multicolumn{4}{|c|}{ 1. July-Aug. 1999} \\
\hline 2451386.2233 & 0.1748 & 3600 & 200 \\
\hline 2451386.2764 & 0.1827 & 5400 & 200 \\
\hline 2451387.2450 & 0.3267 & 5400 & 170 \\
\hline 2451387.3096 & 0.3363 & 5400 & 180 \\
\hline 2451388.2278 & 0.4729 & 5400 & 150 \\
\hline 2451388.2946 & 0.4828 & 5400 & 120 \\
\hline 2451389.2245 & 0.6211 & 5400 & 120 \\
\hline 2451389.2937 & 0.6314 & 3600 & 120 \\
\hline 2451391.1983 & 0.9146 & 3600 & 160 \\
\hline 2451392.2233 & 0.0671 & 5400 & 140 \\
\hline 2451392.2681 & 0.0737 & 1800 & 140 \\
\hline 2451392.3010 & 0.0786 & 3600 & 200 \\
\hline \multicolumn{4}{|l|}{ 2. Feb. 2000} \\
\hline 2451593.9570 & 0.0676 & 2400 & 170 \\
\hline 2451594.9589 & 0.2166 & 2400 & 160 \\
\hline 2451595.9625 & 0.3659 & 3600 & 85 \\
\hline 2451596.9567 & 0.5137 & 3600 & 120 \\
\hline 2451597.9602 & 0.6629 & 3000 & 170 \\
\hline 2451598.9557 & 0.8110 & 3000 & 150 \\
\hline \multicolumn{4}{|c|}{ 3. Nov.-Dec. 2001} \\
\hline 2452237.0161 & 0.6993 & 1800 & 160 \\
\hline 2452238.0092 & 0.8470 & 5400 & 160 \\
\hline 2452238.9832 & 0.9918 & 3300 & 190 \\
\hline 2452239.9882 & 0.1413 & 4200 & 160 \\
\hline 2452240.9630 & 0.2862 & 2400 & 160 \\
\hline 2452242.9929 & 0.5881 & 4500 & 190 \\
\hline 2452243.9588 & 0.7317 & 2400 & 200 \\
\hline 2452244.9763 & 0.8831 & 4800 & 190 \\
\hline
\end{tabular}

Before Doppler imaging was carried out, the wavelengths of all spectra used were corrected to the frame of the primary.

\section{Doppler imaging}

In order to obtain the surface maps of II Peg in these three years, we have used Doppler imaging code DoTS (Collier Comeron 1992) to perform image reconstruction. Like most similar studies using Doppler imaging, the FeI6430.844 $\AA$ and CaI6439.075 Å lines were chosen as map lines.

At first, we calculated two atmosphere models corresponding to the temperatures of photosphere and spot by interpolating the Atlas 9 stellar atmosphere models (Kurucz 1993). Then, we built two lookup tables for FeI6430.844 $\AA$ and CaI6439.075 $\AA$ lines to prepare for the code DoTS by using the spectral synthesis package INTENSE (Unruh 1994). In the course of calculating lookup tables, 7 nearby blends of FeI6430.844 $\AA$ line and 6 nearby blends of CaI6439.075 line are included into the spectral synthesis. The atomic line parameters of all elements in spectral synthesis are adopted
Table 2. The physical parameters of II Peg.

\begin{tabular}{ll}
\hline \hline Parameter & Value \\
\hline$T_{\text {photosphere }}$ & $4600 \mathrm{~K}$ \\
$T_{\text {spot }}$ & $3500 \mathrm{~K}$ \\
$\log g$ & 3.2 \\
$v \sin i$ & $23 \mathrm{~km} \mathrm{~s}^{-1}$ \\
Inclination $i$ & $60^{\circ}$ \\
Microturbulence $\xi$ & $2.0 \mathrm{~km} \mathrm{~s}^{-1}$ \\
Macroturbulence $\zeta$ & $4.0 \mathrm{~km} \mathrm{~s}^{-1}$ \\
Iron abundance & $-0.4 \mathrm{dex} \mathrm{solar}$ \\
Calcium abundance & solar \\
\hline
\end{tabular}

Table 3. The reduced $\chi^{2}$ for each result map.

\begin{tabular}{ll}
\hline \hline Map line & $\chi^{2}$ \\
\hline FeI(1999) & 1.23 \\
$\mathrm{CaI}(1999)$ & 1.47 \\
$\mathrm{FeI}+\mathrm{CaI}(1999)$ & 1.47 \\
$\mathrm{FeI}(2000)$ & 0.78 \\
$\mathrm{CaI}(2000)$ & 0.78 \\
$\mathrm{FeI}+\mathrm{CaI}(2000)$ & 0.87 \\
$\mathrm{FeI}(2001)$ & 0.90 \\
$\mathrm{CaI}(2001)$ & 0.88 \\
FeI+CaI(2001) & 0.90 \\
\hline
\end{tabular}

from Strassmeier's (1997), Strassmeier et al.'s (1997), and Strassmeier \& Bartus's (2000) results, which were checked by using the observed spectra of the Sun and Arcturus. In addition, the instrument profile is also counted into the spectral synthesis. The physical parameters of II Peg are listed in Table 2, most of them are adopted from Berdyugina et al.'s (1998b) results.

For the observational data in each observing season, at first, we carried out Doppler imaging using the code DoTS for each line independently, and then we preformed image reconstruction using the two lines simutaneously. To do so, we can compare the result maps from single lines with the ones from two lines. On 25 Nov. 2001, unfortunately, a cosmic ray hit inside the FeI6430.844 A profile of the II Peg spectrum, so the profile at this phase $(0.9918)$ is not included in the image reconstruction using the FeI6430.844 $\AA$ line independently and using the FeI6430.844 $\AA$ and CaI6439.075 A lines simultaneously.

In the course of image reconstruction, we used reduced $\chi^{2}$ statistics (Collier Comeron 1995) to quantify the match between the synthetic profile and the observational profile, and adopted the criterion of Collier Comeron (1995) and Unruh et al. (1995) to determine when the iteration should be stopped. The final reduced $\chi^{2}$ for each result map is listed in Table 3 .

Figure 1 shows the maps for the 1999 observing season reconstructed using FeI6430.844 $\AA$ and CaI6439.075 A lines, respectively, and Fig. 2 shows the maps of the 2000 observing season for these two lines. The maps of the 2001 observing season for the same lines are displayed in Fig. 3. The maps reconstructed by using FeI6430.844 $\AA$ and CaI6439.075 $\AA$ lines 

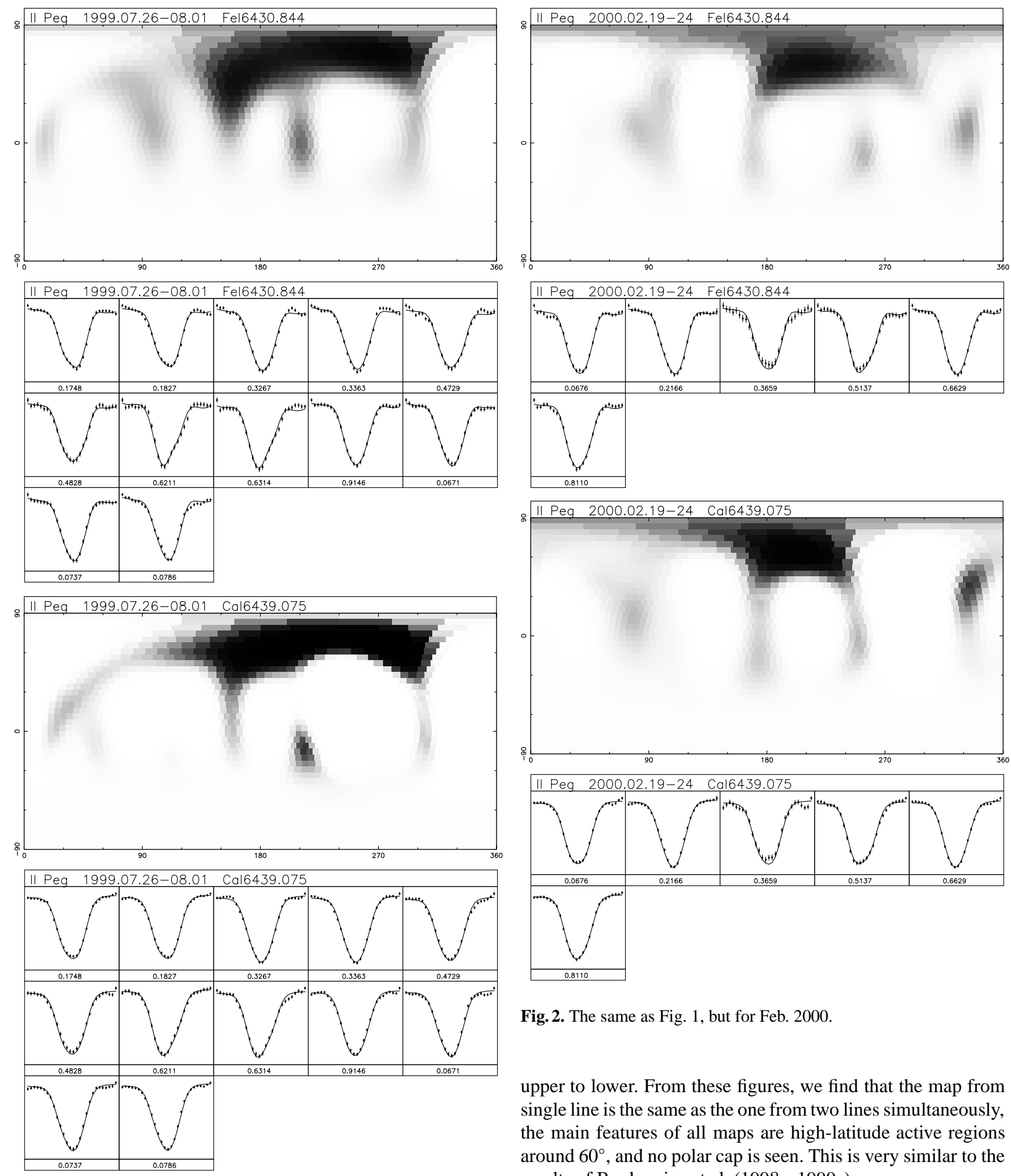

Fig. 1. The Doppler imaging of II Peg from lines FeI6430.844 $\AA$ and CaI6439.075 Å respectively in July-Aug. 1999.

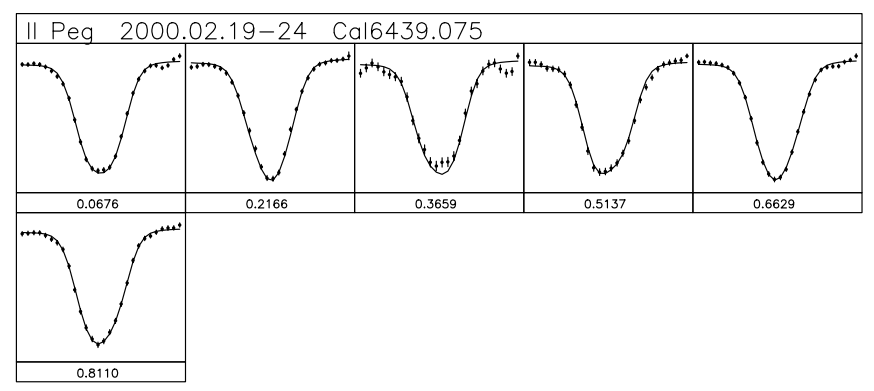

Fig. 2. The same as Fig. 1, but for Feb. 2000.

upper to lower. From these figures, we find that the map from single line is the same as the one from two lines simultaneously, the main features of all maps are high-latitude active regions around $60^{\circ}$, and no polar cap is seen. This is very similar to the results of Berdyugina et al. (1998a, 1999a).

Because no significant differences between the maps from single lines and the one from two lines are found, from now on, we only discuss the results with the aid of the maps from two lines. In Fig. 4, we can see that the main features of the map from July-Aug. 1999 are two large connected high-latitude active regions, which are located at longitude $190^{\circ}$, latitude $60^{\circ}$, and longitude $270^{\circ}$, latitude $65^{\circ}$. From Fig. 5, it can be found

simultaneously for these three years are shown in Figs. 4-6, where the lower panel is the 3D maps arranged from phase 0.0 to 0.875 with the phase increment 0.125 , from left to right, 

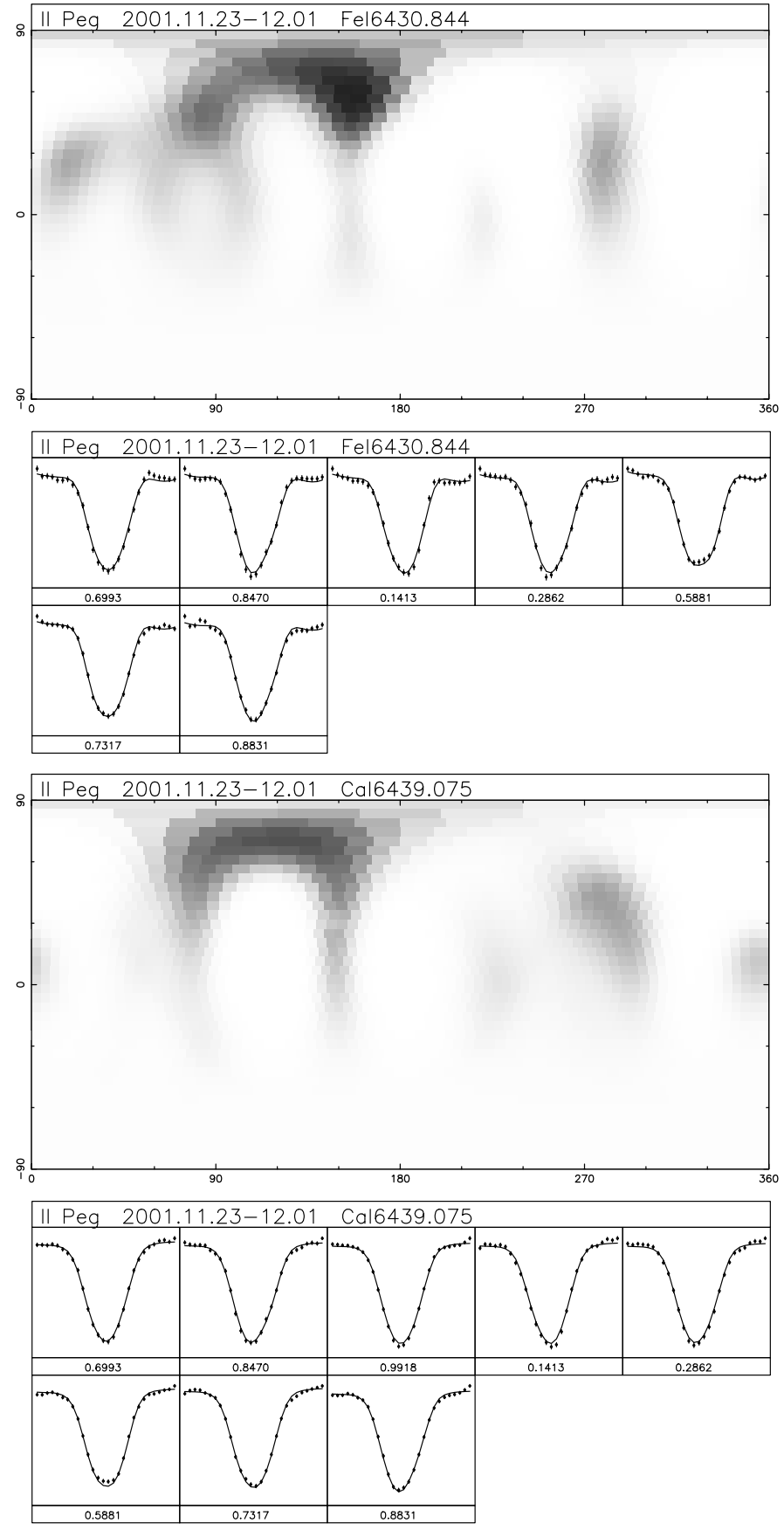

Fig. 3. The same as Fig. 1, but for Nov.-Dec. 2001.

that the main feature of the map in Feb. 2000 is a single large high-latitude spot centered at longitude $210^{\circ}$, latitude $63^{\circ}$. It is also obvious that two small weak spots appear in low-latitude region with longitude $80^{\circ}$, latitude $15^{\circ}$, and longitude $335^{\circ}$, latitude $28^{\circ}$. In Fig. 6, it can be seen that the main features of the map from Nov.-Dec. 2001 are two very close high-latitude spots located at longitude $90^{\circ}$, latitude $62^{\circ}$ and longitude $150^{\circ}$, latitude $62^{\circ}$. Another significant feature is a low-latitude weak spot centered at longitude $280^{\circ}$, latitude $33^{\circ}$. Other small weak spots around the equator may be artifacts and the mirror images of features in high-latitude regions.

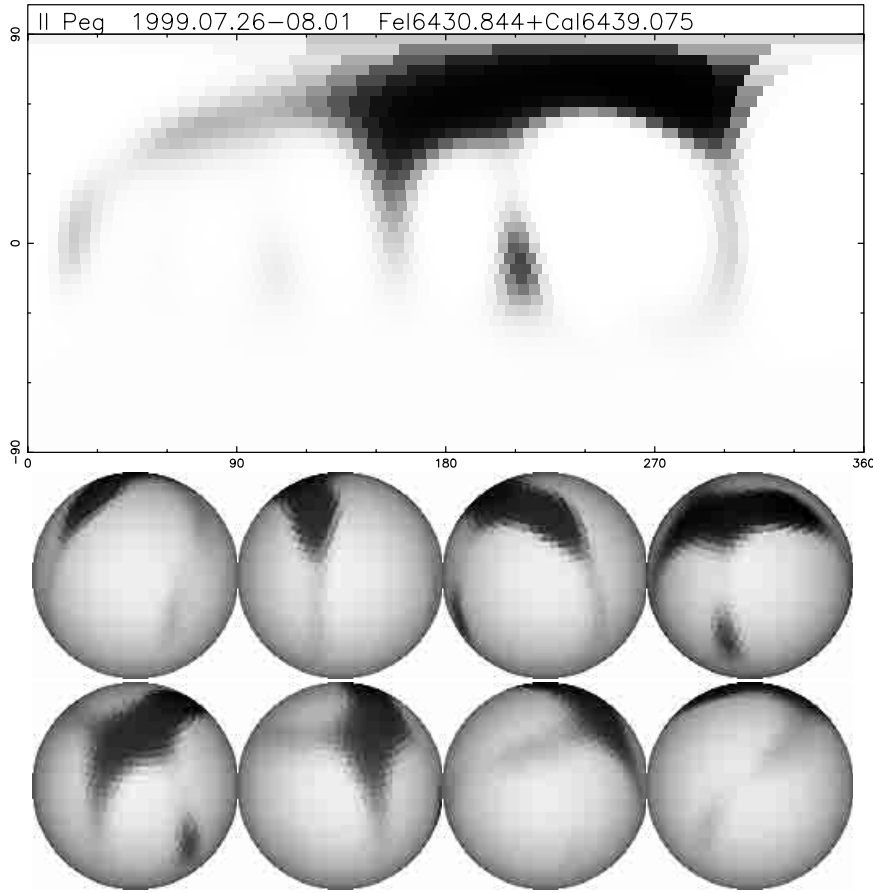

Fig. 4. The Doppler imaging of II Peg using FeI6430.844 $\AA$ and CaI6439.075 Å lines simultaneously in July-Aug. 1999.

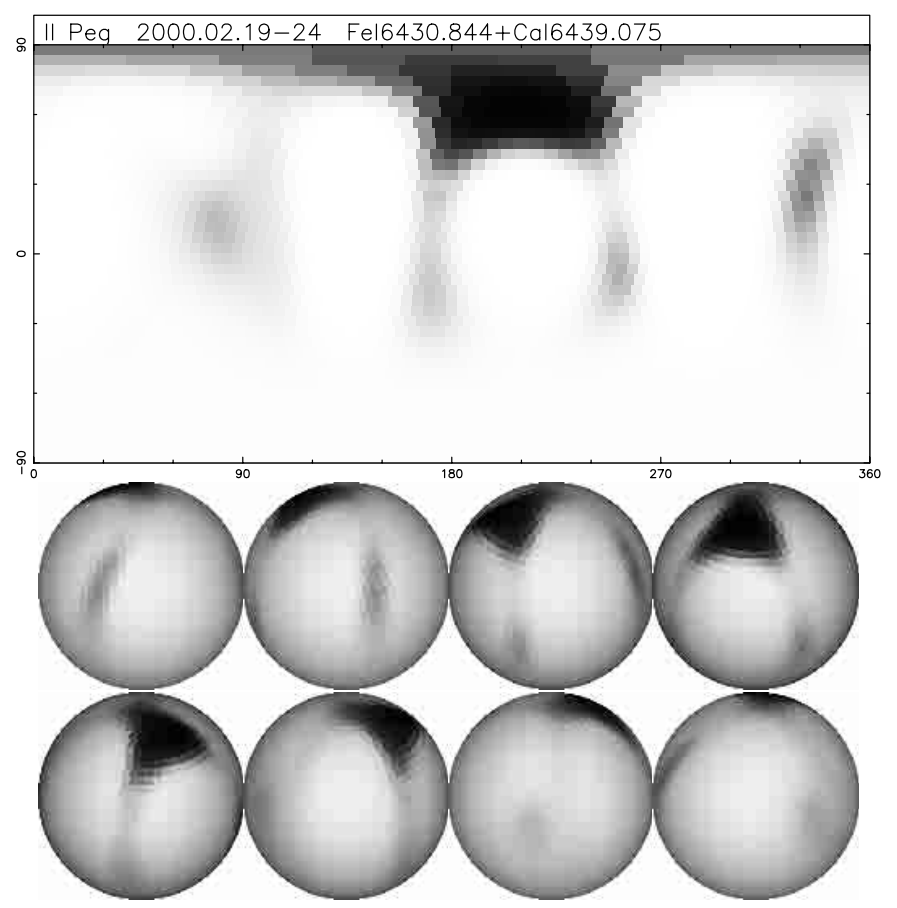

Fig. 5. The same as Fig. 4, but for Feb. 2000.

\section{Discussion}

From our observations, three new surface maps have been derived. In Figs. 4-6 we have found that some large changes in spot distribution, including the position, intensity and size of the spots, are happened during our three observing runs.

In order to compare with previous results of surface imaging, we use Berdyugina et al.'s (1998a, 1999a) idea to trace the spot evolution, and continue to monitor the changes of the spots 


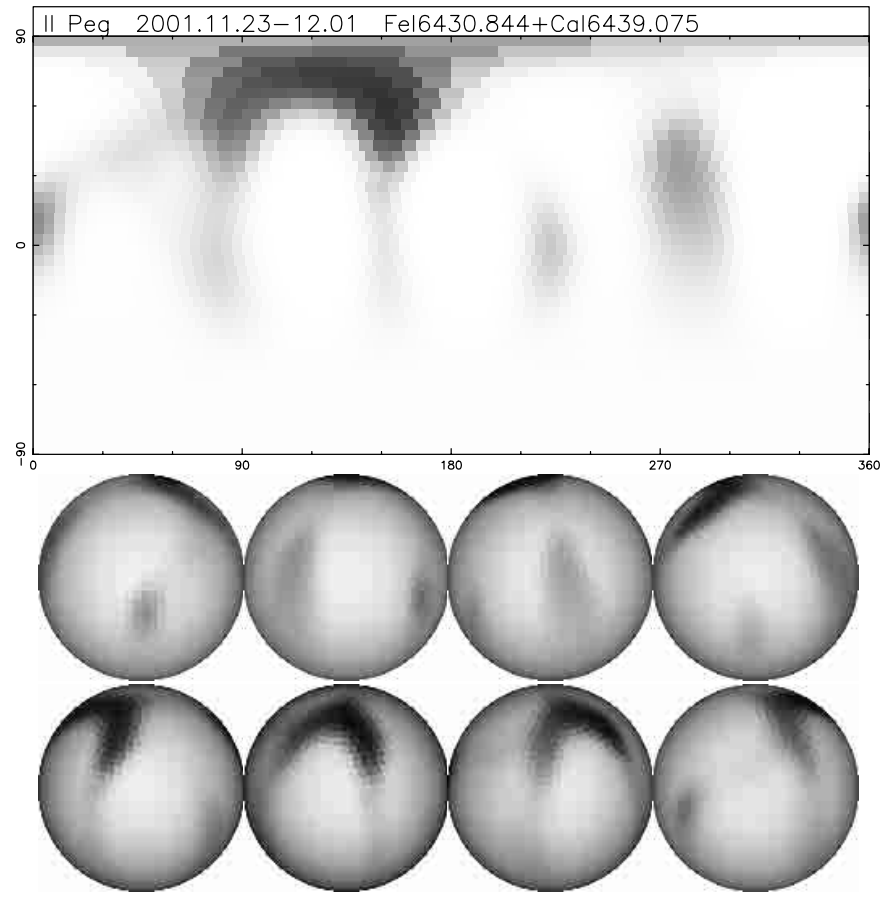

Fig. 6. The same as Fig. 4, but for Nov.-Dec. 2001.

at the active longitude regions. Therefore, only the spots around two predicted active longitude regions separated by about half of the rotational period will be used in the discussion of spot evolution.

According to Berdyugina et al. (1998a), the spot configuration migrates to earlier orbital phases with a mean rate of $0.117^{\circ} /$ day due to the difference between the rotational and orbital periods. In our surface maps, the orbital phase increases from right to left, namely against the direction of the longitude increment. This means that the active longitudes of II Peg migrate to the right in our surface maps. Hence, it can be found that the main connected high-latitude active regions in JulyAug. 1999 become a single large spot in the high-latitude $\left(63^{\circ}\right)$ region and a separated small spot in the low-latitude $\left(28^{\circ}\right)$ region in Feb. 2000. The main high-latitude spot in Feb. 2000 changes to be a low-latitude $\left(33^{\circ}\right)$, small and weak spot in Nov.-Dec. 2001, and the two very close main active regions in Nov.-Dec. 2001 are located in another hemisphere, which may have evolved from two small, weak, low-latidude $\left(28^{\circ}\right.$ and $\left.15^{\circ}\right)$ spots in Feb. 2000. These behaviors obey the longitude evolution rule of Berdyugina et al. (1998a, 1999a), and the position, intensity and size of the spots change.

On the other hand, if we consider the active longitude regions where the main spot features appear, it can be found that the main spot features in 1999 and 2000 are around the same active longitude, and the ones in 2001 are around another active longitude separated by about half of the period of the system from the one of 1999 and 2000. This phenomenon shows that there is a switch between two active longitudes from 2000 to 2001, which is in contradiction to the activity cycle of Berdyugina et al. (1999a). Based on Berdyugina et al.'s (1999a) results, our observing seasons are inside the 7 th halfcycle of II Peg well. Therefore, no switch between active

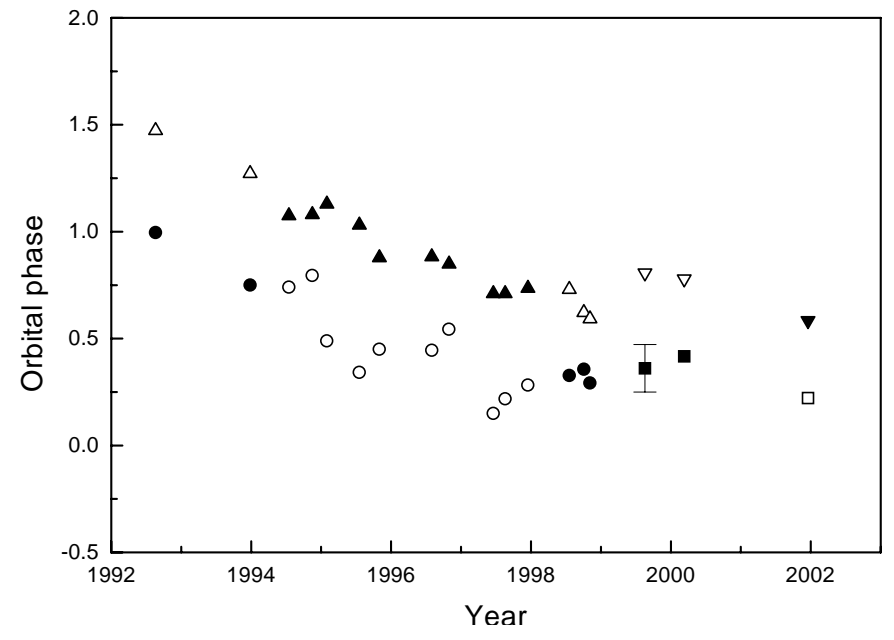

Fig. 7. The spot longitudes of II Peg versus time. Upright triangles and circles present Berdyugina et al.'s data, and inverted triangles and squares present our new data. The solid symbols mean larger active regions, and the open symbols mean smaller active regions.

longitudes should happen. But, from our new results, the switch between two active longitudes occurs from 2000 to 2001. This can be seen in Fig. 7 which includes Berdyugina et al.'s (1999a) and our spot longitude data. In this figure, only the positions of those spots around the active longitudes are plotted, i.e. two large connected high-latitude spots at longitude $190^{\circ}, 270^{\circ}$ and the small spot at longitude $70^{\circ}$ in 1999 , the large highlatitude spot at longitude $210^{\circ}$ and the small low-latitude spot at longitude $80^{\circ}$ in 2000 , the low-latitude weak spot at longitude $280^{\circ}$ and the large high-latitude spot at longitude $150^{\circ}$ in 2001. Because two large high-latitude spots of 1999 are connected and very close to each other in intensity, it is difficult to determine which one has the lower temperature. We choose the average value of the two spot longitudes in Fig. 7, and plot an error bar to represent the uncertainty, which equals the distance between the longitudes of these two spots. As for other spots, the typical error in the longitudes of the spot centers is about $5-10^{\circ}$.

Such an event demonstrates that our results seem to be contrary to the activity cycle derived by Berdyugina et al. (1999a). The next switch predicted by Berdyugina et al.'s (1999a) result between active longitudes will take place in 2003.85, but our observations show a switch in 2001.96, which is off by about two years. Because this activity cycle was originally obtained using long-term photometric data and surface imaging data (Berdyugina \& Tuominen 1998c), it should be reliable. Perhaps the switch detected by us is just an occasional event in an activity cycle of II Peg; we need further observations to clarify this proposition.

In addition, for II Peg, a strong optical flare $(\mathrm{Gu} \&$ Tan 2001) occurred in Sept. 2000. This event may be one of the reasons resulted in the above redistribution of surface spot structure, which drives the spots to change their position, intensity and size. A similar result was also found by Berdyugina et al. (1999b).

From the three maps in 1999-2001, it can be found that the spot evolution seems to follow that the high-latitude main spot 
migrates to the low-latitude region, and becomes weak, while the low-latitude small spot on another hemisphere migrates to a high-latitude region and becomes a large spot.

\section{Summary}

Based on our new observations and analysis, the following results can be derived:

1. The main features of surface maps for 1999-2001 are high-latitude (around $60^{\circ}$ ) active regions. In addition, there are some low-latitude spots in three maps. No polar cap is seen in these maps.

2. During our observing runs, the large connected active regions in 1999 become a single large high-latitude spot and a small, weak low-latitude spot in 2000. The large high-latitude spot in 2000 becomes small and weak in 2001, and migrates to the low-latitude region. The main high-latitude spots in 2001 appear in another active longitude region, which is separated about half of a rotational period from the oringinal active longitude region.

3. The surface patterns in the last two years (2000 and 2001) have changed, including the position, intensity and size of active regions. This may be the result of the strong optical flare that occurred in Sept. 2000, between our two observing runs of 2000 and 2001.

Acknowledgements. This work was supported by the Applied and Basic Research Foundation of Yunnan Province, China. We are very appreciate to Dr. Unruh for sending us a copy of her INTENSE package. Many thanks are given to Dr. Collier Comeron for his kind help when we used the DoTS code. We are very grateful to the anonymous referee for his (her) constructive suggestions and comments on our paper, which lead to a large improvement in our manuscript. We also thank the CCP7 Project for providing the DoTS code.

\section{References}

Berdyugina, S. V., Berdyugin, A. V., Ilyin, I., et al. 1998a, A\&A, 340, 437

Berdyugina, S. V., Jankov, S., Ilyin, I., et al. 1998b, A\&A, 334, 863

Berdyugina, S. V., \& Tuominen, I. 1998c, A\&A, 336, L25

Berdyugina, S. V., Berdyugin, A. V., Ilyin, I., et al. 1999a, A\&A, 350, 626

Berdyugina, S. V., Ilyin, I., \& Tuominen, I. 1999b, A\&A, 349, 863

Collier Cameron, A. 1992, in Surface Inhomogeneities on Late-type Stars, ed. P. B. Byrne, \& D. J. Mullan, 33

Collier Cameron, A. 1995, MNRAS, 275, 534

Gu, S.-H., \& Tan, H.-S. 2001, in 12th Cambridge Workshop on Cool stars, Stellar systems, and the Sun, ASP Conf. Ser., in press

Gu, S.-H., \& Tan, H.-S., \& Shan, H.-G. 2002, in Modelling of Stellar Atmosphere, IAU Symp., 210, in press

Jiang, S.-Y. 1996, in Ground-based Astronomy in Asia, ed. N. Kaifu, 335

Kurucz, R. L. 1993, Atlas 9 CD, No. 13

Montes, D., Fernandez-Figueroa, M. J., De Castro, E., et al. 1997, A\&AS, 125, 263

Rodonò, M., Messina, S., Lanza, A. F., et al. 2000, A\&A, 358, 624

Rucinski, S. M. 1977, PASP, 89, 280

Strassmeier, K. G. 1997, A\&A, 319, 535

Strassmeier, K. G., Hubl, B., \& Rice, J. B. 1997, A\&A, 322, 511

Strassmeier, K. G., \& Bartus, J. 2000, A\&A, 354, 537

Unruh, Y. C. 1994, Ph.D. Thesis, University of Sussex

Unruh, Y. C., Collier Cameron, A., \& Cutispoto, G. 1995, MNRAS, 277,1145

Zhao, G., \& Li , H.-B. 2001, CJAA, 1, 555 